\title{
Tilings of the sphere with right triangles III: the asymptotically obtuse families
}

\author{
Robert J. MacG. Dawson* \\ Department of Mathematics and Computing Science \\ Saint Mary's University \\ Halifax, Nova Scotia, Canada \\ Blair Doyle ${ }^{\dagger}$ \\ HB Studios Multimedia Ltd. \\ Lunenburg, Nova Scotia, Canada B0J 2C0
}

Submitted: Feb 7, 2007; Accepted: Jun 28,2007; Published: Jul 5, 2007

Mathematics Subject Classification: 05B45

\begin{abstract}
Sommerville and Davies classified the spherical triangles that can tile the sphere in an edge-to-edge fashion. However, if the edge-to-edge restriction is relaxed, there are other such triangles; here, we continue the classification of right triangles with this property begun in our earlier papers. We consider six families of triangles classified as "asymptotically obtuse", and show that they contain two non-edge-toedge tiles, one (with angles of $90^{\circ}, 105^{\circ}$ and $45^{\circ}$ ) believed to be previously unknown.
\end{abstract}

Keywords: spherical right triangle, monohedral tiling, non-edge-to-edge, nonnormal, asymptotically obtuse

${ }^{*}$ Supported by a grant from NSERC

${ }^{\dagger}$ Supported in part by an NSERC USRA 


\section{Introduction}

A tiling is called monohedral (or homohedral) if all tiles are congruent, and edge-to-edge (or normal) if two tiles that intersect do so in a single vertex or an entire edge. In 1923, D.M.Y. Sommerville [8] classified the edge-to-edge monohedral tilings of the sphere with isosceles triangles, and those with scalene triangles in which the angles meeting at any one vertex are congruent. H.L. Davies [1] completed the classification of edge-to-edge monohedral tilings by triangles in 1967 (apparently without knowledge of Sommerville's work), allowing any combination of angles at a vertex. Davies' work omitted many details; these were filled in recently by Ueno and Agaoka [9].

Non-edge-to-edge tilings were apparently first considered in [2], where a complete classification of isosceles spherical triangles that tile the sphere was given. In [3], it was shown that, with one exception, every triangle that tiles the sphere but does not do so edge-to-edge has at least one combination of angles other than two right angles that add to $180^{\circ}$. This paper and its companion papers $[4,5,6]$ continue the program of classifying the triangles that tile the sphere. A more complete description of the program is given in $[4]$.

An important and still open problem involving monohedral tilings of the sphere is the question, recorded by Ruziewicz in the "Scottish Book" [7, problem 60] in the late 1930's or early 1940's, of whether such tilings exist with tiles of arbitrarily small diameter. None of the tiles exhibited in this sequence of papers offers any improvement on the $\left(90^{\circ}, 60^{\circ}, 36^{\circ}\right)$ triangle, with diameter $37.3774 \ldots{ }^{\circ}$, which was already known [8] by the time Ruziewicz raised the problem.

In [4] we introduced the idea of the vertex signature $\mathcal{V}_{T}$ of a triangle $T$ with angles $(\alpha, \beta, \gamma)$, defined to be the set of triples $\left\{(a, b, c): a \alpha+b \beta+c \gamma=360^{\circ}\right\}$. Such a vector with $a<2$ will be called reduced; if $b>a, c$ it is a beta source and if $c>a, b$, a gamma source.

It was shown in [4] that, for triangles with $\alpha=90^{\circ}, \beta \geq \gamma$ that tile but do not do so edge-to-edge, the affine hull of this set is always two-dimensional, and we may choose a basis for it consisting of the vectors $\left\{(4,0,0),(a, b, c),\left(a^{\prime}, b^{\prime}, c^{\prime}\right)\right\}$ where the second vector is a reduced $\beta$ source and the third a reduced $\gamma$ source. Conversely, any such triple determined a unique set of angles (not necessarily corresponding to a spherical triangle, or even all positive.)

It should be noted that this is not always true for triangles that tile edge-to-edge. In particular, there is a continuous family of tiles with $\alpha+\beta+\gamma=360^{\circ}$, all of which tile the sphere edge-to-edge with four copies [1]. A typical member of this family has only one vector, $(1,1,1)$, in its vertex signature; however, all three angles of any such triangle must be obtuse.

For any reduced $\beta$ source $(a, b, c)$ and for fixed $a^{\prime}, b^{\prime}$, let $\beta(n)$ and $\gamma(n)$ be the angles determined by the vectors $\left\{(4,0,0),(a, b, c),\left(a^{\prime}, b^{\prime}, n\right)\right\}$. It is easy to see that

$$
\lim _{n \rightarrow \infty} \gamma(n)=0
$$


while

$$
\lim _{n \rightarrow \infty} \beta(n)=\frac{(360-90 a)^{\circ}}{b}
$$

and this is independent of $a^{\prime}$ and $b^{\prime}$ (and, indeed, of $c$ ).

We can thus classify the $\beta$ sources $(a, b, c)$, and the families of triangles with those $\beta$ sources, as asymptotically acute, asymptotically right, or asymptotically obtuse, depending on the limiting value of $\beta(n)$. (There is some overlap between these families, as a triangle may have more than one $\beta$ source in its vertex signature.) It turns out that this classification is useful in characterizing the triangles that tile the sphere; different strategies work for the three types of $\beta$ source. The tiles in asymptotically right families were classified in [4] and [5]. In this paper we classify the tiles in asymptotically obtuse families. We shall include for completeness the isosceles tiles and edge-to-edge tilings already classified in [1], [2], and [8]. For some terminology the reader is referred to our previous paper [4].

\section{Classification of tiles}

Theorem 1 The only right triangles in asymptotically obtuse families that tile the sphere are the $\left(90^{\circ}, 120^{\circ}, 90^{\circ}\right),\left(90^{\circ}, 120^{\circ}, 60^{\circ}\right),\left(90^{\circ}, 90^{\circ}, 90^{\circ}\right),\left(90^{\circ}, 90^{\circ}, 45^{\circ}\right),\left(90^{\circ}, 108^{\circ}, 54^{\circ}\right)$, and $\left(90^{\circ}, 105^{\circ}, 45^{\circ}\right)$ triangles.

The first four tiles (Figure 1) are familiar, three being in the list of Sommerville [8] and all in that of Davies [1]. The first three of these admit only one edge-to-edge tiling, and the fourth has two. All of them admit various non-edge-to-edge tilings; these will be considered briefly in the last section.

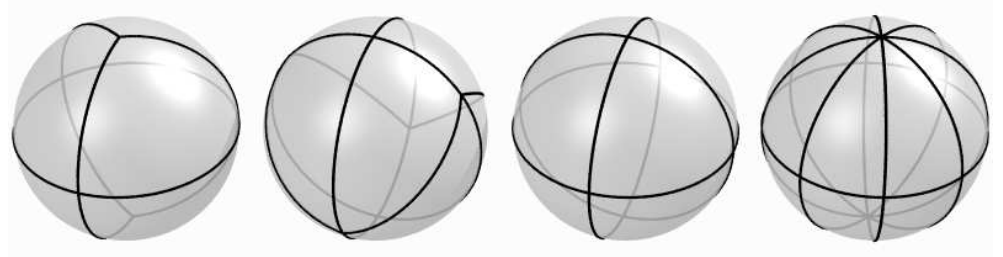

Figure 1: edge-to-edge tilings

The final two triangles tile only in a non-edge-to-edge fashion, with ten and twelve copies respectively. The $\left(90^{\circ}, 108^{\circ}, 54^{\circ}\right)$ triangle was introduced and described in [3]; it admits three different tilings. As will be shown below, the $\left(90^{\circ}, 105^{\circ}, 45^{\circ}\right)$ triangle admits (up to reflection) only one tiling. All af these tilings have chiral symmetry groups; this is common (though not universal) among non-edge-to-edge tilings.

Interestingly, if we extend the edges defining the $45^{\circ}$ angle of the $\left(90^{\circ}, 105^{\circ}, 45^{\circ}\right)$ triangle till they meet again (a construction that also appears in the proof of the next lemma), 

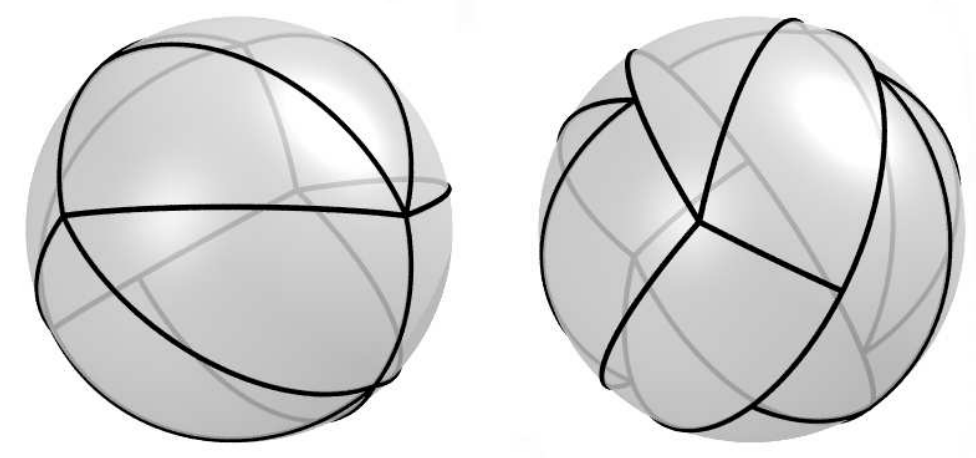

Figure 2: Non-edge-to-edge tilings: $\left(90^{\circ}, 108^{\circ}, 54^{\circ}\right)$, and $\left(90^{\circ}, 105^{\circ}, 45^{\circ}\right)$

the complementary $\left(90^{\circ}, 75^{\circ}, 45^{\circ}\right)$ triangle also tiles the sphere ([2], §3.vii) . There is no obvious relation betwen the two tilings, and the observation is probably best considered as a coincidence.

\section{Proof of Theorem 1}

The proof of Theorem 1 follows, roughly, the pattern established in [4]; we consider each possible reduced $\beta$ source in turn, determine what $\gamma$ sources could work with each, and then classify the tiles (if any) that arise. However, for asymptotically obtuse families, the following lemma makes the process much simpler than was the case there.

Lemma 1 For any right spherical triangle

$$
\gamma \leq \beta<\gamma+90^{\circ}
$$

Proof: The first inequality is by definition and is included only for ease of reference. The second follows by extending the two edges defining the $\gamma$ angle until they meet again, forming a triangle with angles $\left(90^{\circ}, 180^{\circ}-\beta, \gamma\right)$, and noting that the angles of this triangle must add to more than $180^{\circ}$.

Lemma 2 The reduced $\beta$ sources generating asymptotically obtuse families are $(0,3,2)$, $(0,3,1),(0,3,0),(0,2,1),(1,2,1)$ and $(1,2,0)$.

Proof: By definition, $a<2$ and $b>c$. If the right hand side of (2) is obtuse, we have $a+b \leq 3$. Finally, $(0,2,0)$ is ruled out, as we must have $\beta \neq 180^{\circ}$; the remaining possibilities are as listed.

If a $\beta$ source is asymptotically obtuse, it follows from (1) and (2) that (3) is only satisfied for finitely many triangles in that family. (This contrasts with the situation for 
asymptotically right triangles, for which infinite families must often be considered: see $[4,5]$.

The following lemma will be used throughout the proof.

Lemma 3 For every right triangle $T$ in an asymptotically obtuse family that tiles the sphere, one of the following holds:

1. T tiles edge-to-edge (and is listed in [1]);

2. $T$ is the $\left(90^{\circ}, 108^{\circ}, 54^{\circ}\right)$ triangle (given in [3]);

3. $\mathcal{V}_{T}$ contains a reduced vector $V^{\prime}=(0, d, e)$ with both $d$ and e even, and a $\beta$ source $V_{1}$, such that $\left\{(4,0,0), V_{1}, V^{\prime}\right\}$ is a basis for $\mathcal{V}_{T}$.

Proof: Assume that the triangle tiles the sphere but does not tile edge-to-edge. It was proved in [3] that the $\left(90^{\circ}, 108^{\circ}, 54^{\circ}\right)$ triangle is the only such right triangle that has no second split vector. Any other right triangle $T$ thus has a vector $(p, q, r) \in \mathcal{V}_{T}$ that splits. Either $p=0$ or $p=2$. In the first case $V^{\prime}=(0, q, r)$; in the latter case $2(p, q, r)-(4,0,0)=$ $(0,2 q, 2 r)$ is in $\mathcal{V}_{T}$ and we may take this to be $V^{\prime}$.

Any reduced vector $(a, b, c)$ in the affine hull of $(4,0,0)$ and $V^{\prime}$ must be $V^{\prime}$ itself or of the form $\frac{1}{4}(4,0,0)+\frac{3}{4} V^{\prime}$. In the first case $b$ and $c$ would both be even; in the second case, they would be divisible by 3 and $a$ would be greater than 0 . None of the possible reduced $\beta$ sources $V_{1}$ listed in Lemma 2 meet these criteria; thus the three vectors are independent and generate $\mathcal{V}_{T}$.

Note that the $\left(90^{\circ}, 108^{\circ}, 54^{\circ}\right)$ triangle has $\mathcal{V}_{T}=\{(4,0,0),(1,2,1),(1,1,3),(1,0,5)\}$. Only one of these vectors, $(1,2,1)$, is a $\beta$ source; we may thus assume that if a triangle $T$ does not have this $\beta$ source, it tiles edge-to-edge or has a reduced split vector.

Proposition 1 The only right triangle with $V_{1}=(0,3,2)$ that tiles the sphere is the $\left(90^{\circ}, 90^{\circ}, 45^{\circ}\right)$ triangle.

Proof: From Davies' list [1] or from corollary 3 of [9] we can establish that the $\left(90^{\circ}, 90^{\circ}, 45^{\circ}\right)$ triangle is the only triangle in this family that tiles normally; and the $\left(90^{\circ}, 108^{\circ}, 54^{\circ}\right)$ triangle is not in the family. Thus, any other tile would, by the preceding lemma, have to have a reduced vector $V=(0, d, e)$ with $d, e$ even.

If $d \geq 8$ we have $\gamma \leq \beta \leq 45^{\circ}$ and the triangle is not spherical. If $d=6$ we clearly have $e<2$ (hence 0 ), $\beta=60^{\circ}$, and $\gamma=90^{\circ}$; but this contradicts our assumption that $\beta \geq \gamma$. For $d=4$ we have again $e=0$, and we get the $\left(90^{\circ}, 90^{\circ}, 45^{\circ}\right)$ triangle, discussed above.

When $d=2, \gamma=\frac{360^{\circ}}{3 e-4}, \beta=\frac{360(e-2)^{\circ}}{3 e-4}$, and $\beta-\gamma=\frac{360(e-3)^{\circ}}{3 e-4}$ which satisfies (3) only for $3 \leq e<8$. Of the two $V$ consistent with this, $(0,2,4)$ gives the $\left(90^{\circ}, 90^{\circ}, 45^{\circ}\right)$ triangle again, and $(0,2,6)$ gives the $\left(90^{\circ}, 102 \frac{6}{7}^{\circ}, 25 \frac{5}{7}^{\circ}\right)$ triangle whose area does not divide that of the sphere. 
If $d=0$, we have $\gamma=\frac{360^{\circ}}{e}$ and thus $\beta=\frac{360(e-2)^{\circ}}{3 e}$. These satisfy (3) only for $5 \leq e<20$; and the excess $\alpha+\beta+\gamma-180^{\circ}$ divides $720^{\circ}$ only when $e+4 \mid 96$. This gives a solution only for $e=8$ or 12 . In the first case we obtain the $\left(90^{\circ}, 90^{\circ}, 45^{\circ}\right)$ triangle once more. For $e=12$ we get the $\left(90^{\circ}, 100^{\circ}, 30^{\circ}\right)$ triangle, which has $\mathcal{V}_{T}=$ $\{(4,0,0),(3,0,3),(2,0,6),(1,0,9),(0,3,2),(0,0,12)\}$. This has $(0,3,2)$ as its only $\beta$ source and no $\beta$ split; and the $\gamma$ splits have at least three $\gamma$ angles.

For there to be as many $\beta$ angles as $\gamma$ angles, there must exist a $(0,3,2)$ vertex. All five of the angles at such a vertex have hypotenuse edges; at least one of these five edges must be unpaired, and in the absence of $\beta$ splits such an unpaired edge must have its $\beta$ end at the $(0,3,2)$ vertex $(A$ in Figure 3$)$. The other end is a $\gamma$ split with at least 3 excess $\gamma$ angles $(B$,in Fig. 3) The $\gamma$ angle on the unpaired edge must be adjacent to the overhang at $B$, so at most two $(0,3,2)$ vertices can share a split. (In fact, it may be shown that no sharing is possible; but we do not need this.) Associating one or two $(0,3,2)$ vertices with at least three additional $\gamma$ angles gives an overall excess of $\gamma$ angles in the supposed tiling.

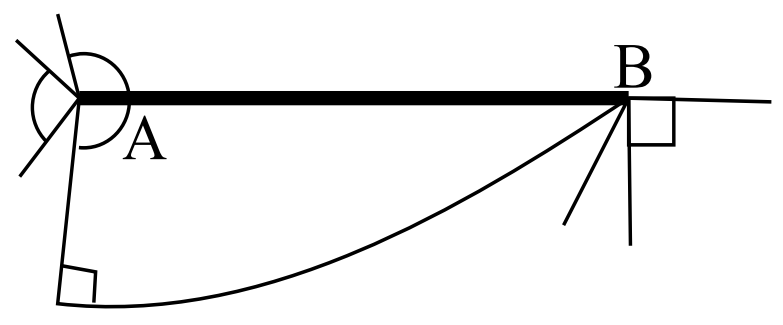

Figure 3: Configuration at a $(0,3,2)$ vertex

Proposition 2 The only right triangles with $V_{1}=(0,3,1)$ that tile the sphere are the $\left(90^{\circ}, 90^{\circ}, 90^{\circ}\right)$ triangle and the $\left(90^{\circ}, 105^{\circ}, 45^{\circ}\right)$ triangle.

Proof: The $\left(90^{\circ}, 90^{\circ}, 90^{\circ}\right)$ triangle tiles edge-to-edge; as before, any other tile in this family must have a reduced vertex vector $V=(0, d, e)$ with $d, e$ both even. For $d>4$ we can never have $\beta \geq \gamma$. When $d=4$ we can only have $\beta \geq \gamma$ for $e=0$, when we get the $\left(90^{\circ}, 90^{\circ}, 90^{\circ}\right)$ triangle.

When $d=2, \beta=\frac{360(e-1)^{\circ}}{3 e-2}$ and $\gamma=\frac{360^{\circ}}{3 e-2}$. This satisfies (3) only for $e \leq 4$; when $e=4$ the excess does not divide $720^{\circ}$ and when $e=2$ we get the $\left(90^{\circ}, 90^{\circ}, 90^{\circ}\right)$ triangle again..

Finally, when $d=0$, we get $\gamma=\frac{360^{\circ}}{e}$ and $\beta=\frac{120(e-1)^{\circ}}{e}$. The area of such a triangle divides that of the sphere only when $e+8 \mid 192$, and (3) is satisfied when $4 \leq e \leq 16$. Together, these rule out every triangle except the $\left(90^{\circ}, 105^{\circ}, 45^{\circ}\right)$ triangle when $e=8$.

Proposition 3 The only right triangles with $V_{1}=(0,3,0)$ that tile the sphere are the $\left(90^{\circ}, 120^{\circ}, 60^{\circ}\right)$ triangle and the $\left(90^{\circ}, 120^{\circ}, 90^{\circ}\right)$ triangle. 
Proof: The two triangles given tile edge-to-edge; any other tile would have to have a reduced vertex vector $V=(0, d, e)$ with $d$, e both even. We have $\beta=120^{\circ}$, so $d<3$. For $d=2,(3)$ is only satisfied when $e=2$, which gives the $\left(90^{\circ}, 120^{\circ}, 60^{\circ}\right)$ triangle. For $d=0$ we have $\gamma=\frac{360^{\circ}}{e}$, satisfying (3) if $4 \leq e \leq 10$. For $e=4$ and 6 we get the tiles already mentioned; and when $e=8$ or 10 the area does not divide that of the sphere.

Proposition 4 No right triangle with $V_{1}=(1,2,0)$ tiles the sphere.

Proof: We have $\beta=135^{\circ}$; this allows all normal tiles to be ruled out. Any tile would thus have to have a vertex vector $(0, d, e)$ with $d, e$ even, and evidently $d<3$. When $d=2$ the inequalities (3) are never satisfied. When $d=0$ they are satisfied only for $e=4$ or 6 , and in neither case does the area of the triangle divide that of the sphere.

Proposition 5 The only right triangles with $V_{1}=(1,2,1)$ that tile the sphere are the $\left(90^{\circ}, 90^{\circ}, 90^{\circ}\right)$ and $\left(90^{\circ}, 108^{\circ}, 54^{\circ}\right)$ triangles.

Proof: The triangles mentioned are known to tile [1,3]; any other tile would have a vertex vector $(0, d, e)$ with $d$ and $e$ even. We cannot satisfy (3) for $d>4$; and when $d=4$ or $d=2$ those inequalities are satisfied only by vectors yielding the $\left(90^{\circ}, 90^{\circ}, 90^{\circ}\right)$ triangle. When $d=0$ they are satisfied for $4 \leq e<12$; but the only $e$ for which the area of the triangle divides that of the sphere is 4 , yielding the $\left(90^{\circ}, 90^{\circ}, 90^{\circ}\right)$ triangle once more.

Proposition 6 No right triangle with $V_{1}=(0,2,1)$ tiles the sphere.

Proof: No tile listed in [1] or [3] is in this family, so once more we may assume a vertex vector with $d$ and $e$ even. It is easily seen that $d=0$, and (3) is only satisfied for $e=4$; the area of the resulting $\left(90^{\circ}, 135^{\circ}, 90^{\circ}\right)$ triangle does not divide that of the sphere.

\section{Classification of tilings}

Some tiles yield more than one tiling. Davies [1] classified all normal tilings (and more details were given by Ueno and Agaoka [9]).

We will briefly examine the non-normal tilings with triangles that also tile edge-toedge. We note that when a tiling has a subset such that the symmetry group $G$ of the subset is a proper subgroup of the symmetry group $H$ of its union, the action of $H / G$ on that subset of the tiling generally yields a new tiling.

- The $\left(90^{\circ}, 90^{\circ}, 90^{\circ}\right)$ and $\left(90^{\circ}, 120^{\circ}, 90^{\circ}\right)$ triangles have continuous families of tilings, derived from those in Figure 1 by rotating one hemisphere relative to the other, and no other tilings. 
- The $\left(90^{\circ}, 120^{\circ}, 60^{\circ}\right)$ triangle tiles the hemisphere in three distinct ways, two of these with a left-handed and right-handed form. Up to reflection, then, there are nine distinct continuous families of tilings (with some special cases in common). There are also two distinct tilings without a great circle in the union of the tile boundaries.

- For the $\left(90^{\circ}, 90^{\circ}, 45^{\circ}\right)$ triangle, there are 252 essentially distinct tilings obtained by subdividing the edge-to-edge tiling with $\left(90^{\circ}, 90^{\circ}, 90^{\circ}\right)$ triangles [2]. Each of these yields three continuous families of tilings, not necessarily distinct. There are also other tilings, including some with no great circle in the union of the boundaries. We conjecture that any tiling with this tile can be obtained from any other by repeated rotation of subsets with symmetric union - a process reminiscent of Rubik's Cube, though perhaps impractical to realize mechanically as a puzzle.

It was shown in [3] that the $\left(90^{\circ}, 108^{\circ}, 54^{\circ}\right)$ triangle tiles in three essentially different ways, none edge-to-edge.

Proposition 7 The $\left(90^{\circ}, 105^{\circ}, 45^{\circ}\right)$ triangle admits only one tiling (up to reflection).

Proof: For this triangle, $\mathcal{V}_{T}=\{(4,0,0),(3,0,2),(2,0,4),(1,0,6),(0,3,1),(0,0,8)\}$. The only $\beta$ source is $(0,3,1)$, which must therefore appear in any tiling; and there are no splits involving $\beta$ angles. At a $(0,3,1)$ vertex $(O$ in Figure $4 a)$, then, the hypotenuse of the triangle (1) contributing the $\gamma$ angle must be paired, to avoid a $\beta$ split at the other end $(A)$. Triangle 2 is thus as shown.
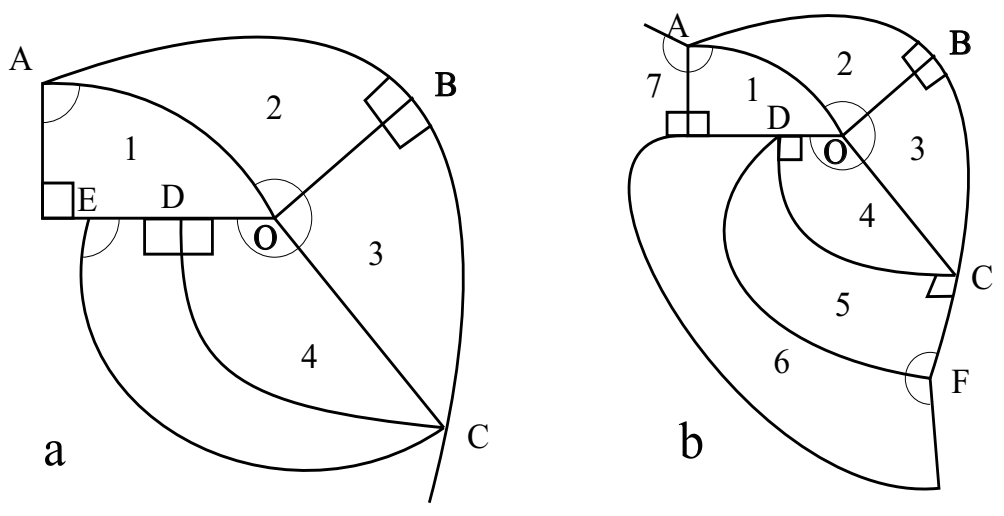

Figure 4: Tiling with the $\left(90^{\circ}, 105^{\circ}, 45^{\circ}\right)$ triangle

As the vertex $A$ can have neither a right angle nor another $\gamma$, the edge $\overline{A B}$ cannnot be matched and there is an overhang at $B$. Triangle 3 is forced; by the same argument there is an overhang at $C$ and we have triangle 4 .

The edge $\overline{C D}$ cannot be covered as in Figure $4 a$, as that would require a $\beta$ split at E. We thus have triangles 5,6 and 7 as shown in Figure $4 b$. Repeating this configuration at $A$ and $F$ gives the entire tiling, as shown in Figure 2. 


\section{Acknowledgement}

We would like to thank the referee for helpful and constructive comments.

\section{References}

[1] Davies, H.L., Packings of spherical triangles and tetrahedra. Proc. Colloquium on Convexity (Copenhagen, 1965) 42-51

[2] Dawson, R. J. MacG., Tilings of the Sphere with Isosceles Triangles. Disc. and Comp. Geom. 30 (2003) 467-487

[3] Dawson, R. J. MacG., Single-split tilings of the sphere with right triangles in Discrete Geometry: In Honor of W. Kuperberg's 60th Birthday (A. Bezdek, ed.; Feb. 2003, Dekker Lecture Notes in Pure and Applied Mathematics), 207-214

[4] Dawson, R. J. MacG. and Doyle, B., Tilings of the sphere with right triangles I: the asymptotically right families, Electronic Journal of Combinatorics 13(1) (2006) \#R48

[5] Dawson, R. J. MacG. and Doyle, B., Tilings of the sphere with right triangles II: the $(1,3,2),(0,2, n)$ subfamily, Electronic Journal of Combinatorics 13(1) (2006) \#R49

[6] Dawson, R. J. MacG. and Doyle, B., Tilings of the sphere with right triangles IV: the asymptotically acute families, in preparation

[7] Mauldin, R. D. (ed), The Scottish Book, Mathematics from the Scottish Cafe (Secaucus, 1982)

[8] Sommerville, D.M.Y., Division of space by congruent triangles and tetrahedra, Proc. Roy. Soc. Edinburgh 43 (1923), 85-116

[9] Ueno, Y.; Agaoka, Y., Classification of tilings of the 2-dimensional sphere by congruent triangles, Hiroshima Math. J. 32 (2002) 463-540 